\title{
SISTEMAS DE INDICADORES DE SOSTENIBILIDAD Y DESARROLLO TURÍSTICO. APLICACIÓN A LA COMARCA DEL BAJO SEGURA (ALICANTE) ${ }^{1}$
}

\author{
Cipriano Juárez Sánchez-Rubio \\ Departamento de Análisis Geográfico Regional \\ Universidad de Alicante
}

\begin{abstract}
RESUMEN
La zona litoral de la comarca del Bajo Segura ha experimentado una profunda transformación económica, demográfica y paisajística desde 1960 a la actualidad por la masiva afluencia de residentes y turistas en busca de sol, playas e inviernos de suaves temperaturas. La confirmación estadística de este proceso se comprueba mediante el valor de cualquiera de los indicadores utilizados: participación en el producto interior bruto (PIB), empleo generado, renta familiar disponible (RFD), aumento de la población y elevado grado de concentración.

La nueva orientación económica comportaba aumentar la dotación de recursos de agua para garantizar la puesta en valor del territorio. La construcción del trasvase TajoSegura, conformado sobre la base de conseguir la mayor productividad agrícola, es la infraestructura que permite asegurar también el suministro urbano creciente. La concentración de infraestructuras, consumo de agua, capacidad de depósitos, eficiencia en la red de distribución, caudal depurado y sus nuevos aprovechamientos en el espacio litoral mejora la competitividad territorial e individualiza a la zona como un área de privilegio en el general desajuste del crecimiento económico y sostenibilidad del desarrollo urbano-turístico.
\end{abstract}

Palabras clave: sistemas de indicadores, sostenibilidad, desarrollo turístico, déficit hídrico, infraestructuras y calidad del agua.

1 Este trabajo se inscribe en el marco del proyecto de Investigación Planificación y Gestión Sostenible del Turismo. Propuesta Metodológica y aplicación de un SIG (METASIG), con referencia 1FD97-0403, financiado por la Comisión Europea Interministerial de Ciencia y Tecnología (CYCYT) y la Comisión Europea (FEDER), que se desarrolla en la Universidad de Alicante, con participación de investigadores de las Universidades de Santiago de Compostela y Almería y del CSIC. 


\begin{abstract}
Due to the massive arrival of residents and tourists in search of sun, beaches and mild winters, the coastal part of the Vega Baja region, also known as Bajo Segura, has experienced a deep transformation of its economy, demography and landscape between 1960 and the present day. The statistical confirmation of this process can be found in the value of any of the indicators used: participation in the gross domestic product (GDP), employment generated, available household income (AHI), population growth and high concentration degree.

The new economic orientation involved increasing the availability of water resources to guarantee the revaluation of the territory. The construction of the Tajo-Segura transfer, based on the objective of achieving higher agricultural productivity, is the infrastructure that makes it also possible to ensure the growing urban supply. Infrastructure concentration, water consumption, reservoir capacity, efficiency in the distribution network, volume of water treated and its new uses in the coastal spaces improve territorial competitiveness and isolate this region as a privileged area within the general context of imbalances regarding economic growth and urban-tourist development sustainability.
\end{abstract}

Key words: indicator systems, sustainability, tourist development, water deficit, infrastructures and water quality.

\title{
Objetivo y método
}

El objetivo de este trabajo es proponer la utilización de una serie de indicadores hídricos alternativos capaces de medir estadísticamente un aspecto significativo del desarrollo sostenible porque los parámetros tradicionales del desarrollo económico no son adecuados para reflejar sus condiciones. Se trata de mejorar la gestión del agua en los espacios turísticos y hacerlos más compatibles con el medio ambiente. Su valoración proporciona una información veraz y sólida para la toma de decisiones a diferentes niveles, contribuyendo a una sostenibilidad integrada de desarrollo económico, social y medioambiental. Una forma más práctica y conveniente de analizar el proceso del desarrollo turístico y sus implicaciones.

La metodología empleada es la confrontación de los valores estadísticos de una serie de indicadores de Estado-Calidad, Presión y Respuesta de los municipios litorales y de segunda línea referidos a la cantidad y calidad del agua utilizada y su valoración en términos ambientales durante el período de 1991-2000.

La información utilizada procede de diferentes fuentes: $1^{\circ}$ ) La Mancomunidad de los Canales del Taibilla facilita el consumo mensual de cada uno de los diferentes municipios de la comarca durante el período de 1991-2000, 2ํㅜ) Entrevista realizada al grupo rector de las empresas que abastecen en baja a las diferentes entidades locales de la comarca, $3^{\circ}$ ) Cuestionario cumplimentado en su totalidad por los gestores del abastecimiento urbano en baja, 4⿳⺈⿴囗十一) Trabajo de campo en los municipios turísticos para identificar los puntos de instalación de nuevos depósitos de agua, depuradoras, tipo de tratamiento, como el destino del efluente hacia un aumento sostenido de la ampliación de la superficie de regadío, campos de golf, parques y jardines y excedentes vertidos al río Segura. 


\section{Un medio físico-ecológico con riesgos y oportunidades para la expansión del desa- rrollo turístico-residencial}

La actividad turística de los municipios del litoral de la comarca: Guardamar del Segura, Orihuela, Pilar de la Horadada y Torrevieja como los de la segunda línea: Rojales, San Fulgencio y San Miguel de Salinas, se ha desarrollado por unas favorables condicionales naturales como: inviernos benignos, suaves temperaturas a lo largo del año, cielos despejados, abundancia de playas de arena y agradables paisaje (Fig. $\mathrm{n}^{\mathrm{o}}$ 1). Todos son factores de desarrollo turístico que han provocado una masiva afluencia de población estacional y residentes en busca de sol, playas y condiciones climáticas privilegiadas. Por ello, desde los años sesenta se inicia un turismo de alta calidad en la zona litoral del municipio de Orihuela que ha impulsado el desarrollo posterior de mayor rentabilidad económica que la transformación de la superficie agrícola de secano a regadío. Como la cantidad de agua siempre ha sido escasa, los pozos explotados se pusieron al servicio de las urbanizaciones frente al espacio regado, llegando a desaparecer, en algún caso, el terrazgo transformado.

Estas favorables condiciones de clima y costa de gran valor natural que garantizan la oferta turística de calidad, no son capaces de explicar por sí solas la expansión del fenómeno turístico. Los hechos humanos, como la política económica, el desarrollo de las infraestructuras conectando el territorio (ejes viarios), el aumento de la accesibilidad y la dotación de recursos hídricos y energéticos, son tan determinantes o más.

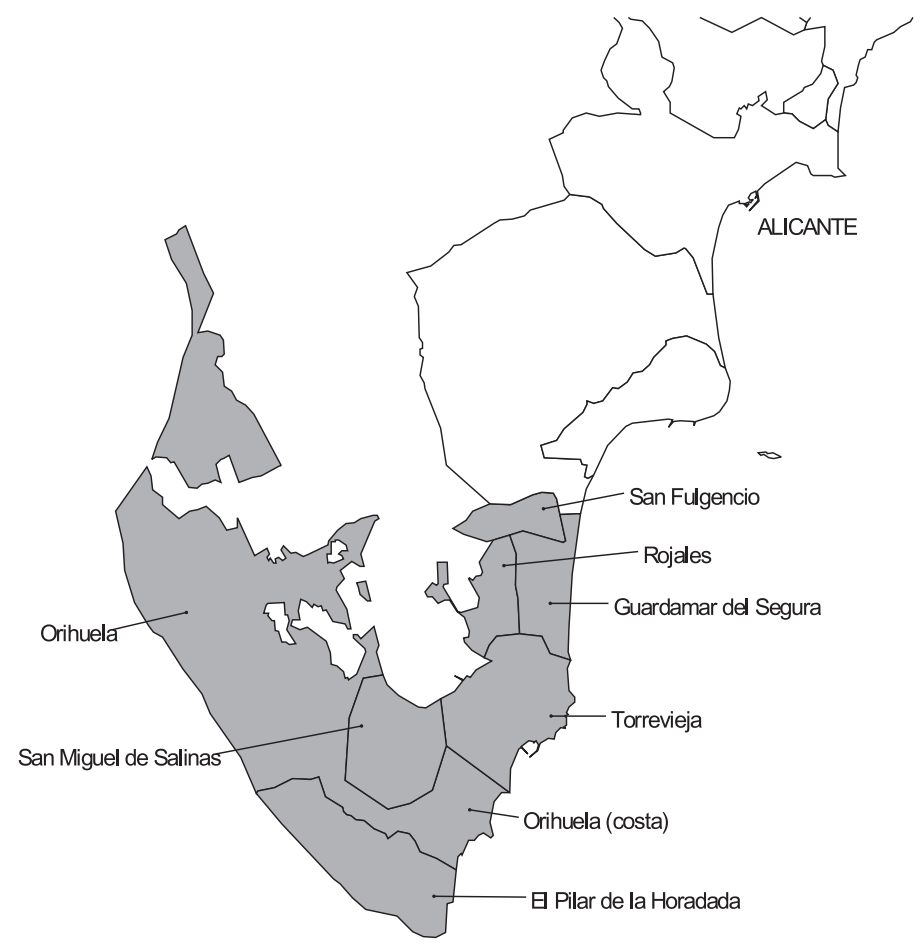

Figura 1. Municipios turísticos de la comarca de la Vega Baja (Alicante). 
El espacio litoral ofrece posibilidades y plantea inconvenientes para el aprovechamiento del suelo. El mayor riesgo radica en el acusado predominio de una precipitación insuficiente, en la elevada irregularidad interanual y estacional y, sobre todo, en la falta de concordancia de estas y la temperatura. El agua en el sector litoral es un recurso escaso directamente relacionado con su clima de naturaleza árida. La cantidad anual recibida varía entre 250 y 300 milímetros al año. Este volumen es insuficiente en relación con las posibilidades productivas de desarrollo agrícola y expansión urbana-turística. La necesidad de disponer de agua suficiente en cantidad y calidad para no condicionar el desarrollo económico del territorio condujo a la regulación de las aguas superficiales mediante embalses, a la explotación de los recursos subterráneos y al desplazamiento de uso de la agricultura hacia el sector turístico-residencial.

Las posibilidades y condiciones del territorio de la comarca del Bajo Segura han estado siempre vinculadas a la gestión y uso de los recursos naturales. Trasladado al campo del desarrollo económico, la regla general admitida es que la disponibilidad de agua determina la capacidad de crecimiento de los lugares. Por esto, las inversiones en sistemas de regulación de agua para uso agrícola, industrial o de servicios se justifican con base a su impacto positivo sobre el crecimiento económico. En consecuencia, cualquier acción tendente a mejorar la situación socioeconómica de la zona turística-residencial de la comarca debía partir de la evaluación previa de sus recursos hídricos mediante la realización del balance hídrico (Cuadro $\mathrm{n}^{\mathrm{o}}$ 1) para determinar sus fuentes, alcance, confiabilidad y calidad de las posibilidades de su utilización y control. Este punto de partida tiene como objetivo determinar la disponibilidad de suministro de agua, su viabilidad y calidad.

Cuadro 1

BALANCE HÍDRICO DE TORREVIEJA. TIPO CLIMÁTICO: E B' ${ }_{3} D$ A'

\begin{tabular}{|l|c|c|c|c|c|c|c|c|c|c|c|c|c|}
\hline & O & N & D & E & F & M & A & M & J & Jl & A & S & AÑ \\
\hline Temperatura media & 19 & 14 & 12 & 11 & 12 & 13 & 15 & 18 & 22 & 25 & 25 & 23 & 17,5 \\
\hline Evapotranspiración potencial (mm) & 72 & 35 & 25 & 23 & 25 & 37 & 53 & 85 & 123 & 150 & 140 & 112 & 880 \\
\hline Precipitación media (mm) & 44 & 29 & 26 & 25 & 22 & 17 & 22 & 17 & 11 & 2 & 4 & 37 & 256 \\
\hline Variación de la reserva & 0 & 0 & 1 & 2 & -3 & 0 & 0 & 0 & 0 & 0 & 0 & 0 & 0 \\
\hline Reserva & 0 & 0 & 1 & 3 & 0 & 0 & 0 & 0 & 0 & 0 & 0 & 0 & 0 \\
\hline Evapotranspiración real & 44 & 29 & 25 & 23 & 25 & 17 & 22 & 17 & 11 & 2 & 4 & 37 & 256 \\
\hline Déficit de agua & 28 & 6 & 0 & 0 & 0 & 20 & 31 & 68 & 112 & 148 & 136 & 75 & 624 \\
\hline Superavit de agua & 0 & 0 & 0 & 0 & 0 & 0 & 0 & 0 & 0 & 0 & 0 & 0 & 0 \\
\hline
\end{tabular}

Im: -42,5 Ih: Ia: Concentración en verano de la eficacia térmica: 46,9 \%

El balance hídrico es un indicador que refleja la realidad sobre la desigual distribución de los recursos. Aplicada su metodología a la zona litoral confirma la coincidencia de la máxima demanda con la mayor escasez. El territorio acoge también un acuífero con capacidad insuficiente para completar las irregularidades de los caudales procedentes de las precipitaciones. La necesidad de disponer de caudales suficientes para atender la agricultura productiva de regadío y no frenar el desarrollo económico condujo a la explo- 
tación de las aguas subterráneas como alternativa de oferta de agua. Aprovechamiento minero del agua en el espacio litoral y su área de influencia que provoca su agotamiento e impulsa acelerar el proyecto de modificar las grandes diferencias de caudales de las vertientes generales o cuencas hidrográficas.

La maximización de las aguas subterráneas ha supuesto un desequilibrio territorial porque en el espacio litoral aumenta la superficie de regadío intensivo, capitalizado, productivo, de nuevos cultivos con venta preferente al mercado exterior, de elevada rentabilidad económica y generación de empleo. Como estas aguas no estaban reguladas y tampoco el nuevo regadío recibía caudales superficiales porque estaban asignados a superficies concretas, surgen conflictos por la competencia entre los diferentes usos. El aumento de la escasez obliga a considerar los usos alternativos y buscar soluciones entre el aprovechamiento agrícola y el turístico-residencial de mayor valor productivo.

En este nuevo escenario la política española de los años sesenta tenía como objetivo conseguir el mayor crecimiento del producto nacional bruto mediante el mejor aprovechamiento y conservación de los recursos agua y suelo. Esta orientación económica comporta un cambio en el sistema de asignación de recursos de agua para aumentar la puesta en valor del territorio. La agricultura productiva de regadío, el desarrollo industrial y el turismo con su correspondiente ocio y consumo urbano han aumentado la presión sobre los escasos recursos existentes. El riesgo de falta de agua en la comarca, factor limitativo de su desarrollo económico y social, puede ser corregido mediante la alternativa de oferta de nuevos caudales procedentes de cuencas hidrográficas excedentes. Se trata de llevar los recursos hídricos sobrantes de las zonas excedentes a los territorios donde se necesitan para conseguir el mayor crecimiento posible del producto interior bruto, primando el desarrollo económico frente al social. Como los mayores incrementos en la demanda de agua se producen en éste área de climatología benigna, se recurre a trasvasar caudales regulados, excedentes y procedentes de la cabecera del río Tajo a la cuenca del río Segura con base a criterios de finalismo de las producciones y de rentabilidad económica.

La superación del déficit hídrico del espacio litoral como en su área de influencia, amenaza constante del desarrollo económico debido a la aridez, modelo territorial basado en la agricultura intensiva de regadío y en el aumento de la población permanente y estacional, supone convertir el riesgo o estrangulamiento en una gran oportunidad productiva La fachada marítima de la comarca de la Vega Baja y la de las provincias de Murcia y Almería, área de preferente aplicación del agua trasvasada, venía siendo, desde la génesis de la concepción del trasvase Tajo-Segura, una reserva geográfica que interesaba promocionar desde el modelo desarrollista propugnado por el Estado. Razones estrictamente económicas, también políticas, propias del modelo desarrollista y de la situación concreta del sureste peninsular (escasez de recursos de agua en relación con las posibilidades productiva) impulsaron la realización del trasvase Tajo-Segura.

El volumen anual asignado para uso agrario de la Vega Baja es de 127,48 $\mathrm{hm}^{3} /$ año distribuidos de la forma siguiente: Vega Baja, Saladares y Riegos de Levante, $125 \mathrm{hm}^{3}$ y 2,84 $\mathrm{hm}^{3}$ para el municipio de Pilar de la Horadada incluido en el sector del Campo de Cartagena (Murcia). También, la asignación de 110 hm³/año para abastecimiento de los municipios y centros industriales conectados con la Mancomunidad de los Canales del Taibilla (MCT). Nueva oferta de disponibilidad de agua proporcionada por los caudales regulados y excedentes del río Tajo sobre los que descansa la nueva agricultura intensiva y de vanguardia, junto a un proceso industrial más sólido, equilibrado y armónico y, un sector servicios, especialmente turístico residencial en expansión, dotado de una elevada rentabilidad y paralelo grado de bienestar socioeconómico. 


\section{Indicadores hídricos de estado-calidad, presión y respuesta. Un análisis orientado a la gestión sostenible}

\subsection{El paradigma del desarrollo sostenible y los nuevos parámetros de medición signifi- cativa}

La etapa del desarrollo industrial asumía el deterioro del medio natural para conseguir un crecimiento económico sostenido. Esta perspectiva evoluciona en la etapa posindustrial al considerar que la degradación ambiental puede frenar el desarrollo. El cambio producido camina hacia la sostenibilidad. La nueva orientación económica conforma un modelo sobre los principios de que el desarrollo debe ser compatible y respetuoso con el medio ambiente, la utilización de los recursos naturales y el legado a las generaciones futuras. En consecuencia, el crecimiento económico elevado y estable debe corresponderse con un paralelo progreso social y la protección del medio ambiente mediante un uso eficiente de los recursos naturales. El paso de un crecimiento económico a desarrollo sostenible comporta añadir calidad de vida y cumplir el principio de equidad. Por esto, los nuevos indicadores se fundamentan sobre el concepto de medir estadísticamente un aspecto significativo del desarrollo sostenible.

El crecimiento económico continuado, tal como existe en la actualidad en el espacio de litoral y segunda línea de la unidad territorial, no es totalmente compatible con un estricto respeto por el entorno natural. El avance del urbanismo por desarrollo inmobiliario produce un modelo de turismo que se fundamenta en el inmediato beneficio económico frente al propugnado por la sostenibilidad. El desarrollo de la sociedad del ocio y los viajes asocian el turismo con la adquisición de una vivienda en la costa con el doble objetivo de tener un lugar de descanso y un medio eficaz de inversión con elevada rentabilidad económica. El resultado en el sector litoral y en los municipios inmediatos ha sido una ocupación extensiva del territorio asociada a la segunda residencia. Este carácter residencial es de preferente especialización inmobiliaria con viviendas de bajo coste demandada por personas de la tercera edad de lugares diferentes que determina un modelo turístico asentado en la baja dotación hotelera y predomino de la oferta residencial secundaria.

El modelo turístico se fundamenta en la ocupación extensiva del suelo litoral favorecido por la disposición de las infraestructuras viarias que tratan de satisfacer el interés general conectando territorios. Sistema de ejes viarios que contribuyen de manera eficaz al desarrollo económico y social al permitir la aproximación de los centros de producción y consumo. Sin embargo, el fenómeno turístico-residencial penetra cada vez más hacia los municipios del interior de la comarca o segunda línea por la mayor disponibilidad de suelo barato y flexibilidad del planeamiento. Dinámica expansionista del proceso turístico-residencial que empieza a afectar a los municipios de Los Montesinos y Algorfa.

El primero de estos centros urbanos es un lugar recientemente segregado del municipio de Almoradí y de preferente economía agrícola por el uso de las aguas del trasvase TajoSegura que apuesta por la vivienda secundaria y el turismo residencial. Cambio de modelo económico propiciado por el crecimiento de la conurbación de Torrevieja que consagra al sector servicios como el de mayor rentabilidad económica y social. Por esto, la voluntad municipal ha optado por la construcción de vivienda fomentando el crecimiento sostenible. Nueva filosofía que se extiende al municipio de Algorfa al contemplarse la posibilidad de un crecimiento demográfico de 1.900 habitantes a 7.000 personas mediante un proyecto de viviendas residenciales.

Este avance progresivo del proceso urbanizador difundido desde el sector marítimo comporta la necesidad de racionalizar su ordenación territorial. Y es que, existen una serie 
de entidades locales entre cuyos núcleos urbanos se generan vinculaciones económicas y sociales, especializaciones e interdependencias que superan el límite municipal y hacen necesaria la planificación conjunta y la coordinación de determinados servicios y obras. Por esto, los municipios de Almoradí y Algorfa han pedido la adhesión de Torrevieja y Orihuela para la constitución del área metropolitana de la Vega Baja. Entidad administrativa supramunicipal reconocida por la Generalidad Valenciana, mediante la promulgación de la Ley de Áreas Metropolitanas de 17 de mayo de 2001 que posibilitaría la ordenación del crecimiento urbanístico-residencial. Sector turístico y de ocio que apoyado en las nuevas infraestructuras de disponibilidad de agua y efecto del AVE garantizan un nuevo proceso de expansión.

La confirmación estadística del crecimiento del fenómeno turístico-residencial de los siete municipios considerados se realiza mediante el gran aumento de la población censada entre los años 1960 y 2001. Se ha pasado de 66.759 (100\%) habitantes a 144.392, lo que en términos relativos significa que la población ha crecido un $216 \%$. Esta dinámica persiste el tiempo y se comprueba cuando se toma como referencia la población empadronada al 1/1/ 03. En el corto período de tiempo del bienio 2001-2003 se pasa de aquella cantidad a 193.933 habitantes, equivalente al 290\%. Es decir, en algo más de 40 años la población de los municipios turísticos casi se triplica frente al desigual comportamiento y reducido crecimiento (sólo el $129 \%$ ) del resto de la comarca al pasar de 66.697 habitante en 1960 a 86.651 en el recuento de 2001. Dinámica demográfica que guarda una paralela correspondencia con la evolución económica ya que los niveles de renta más bajo coinciden con las poblaciones de marcado carácter agrícola, la mayoría enclavada en el territorio interior de la comarca, elevándose los índices de riqueza conforme más próximo se está del litoral. Parámetro económico de la Renta Familiar Disponible que jerarquiza a los municipios de Guardamar y Torrevieja como los dos primeros lugares en la comarca desde el año 1981 al 2002.

El turista característico de los años sesenta confesaba venir a los municipios del litoral motivado por el sol, clima, temperatura, entorno y paisaje. Algo menos por el ambiente, el baño y las actividades al aire libre. Esta concepción empieza a cambiar a partir de los años ochenta debido a que las sociedades desarrolladas consideran que el medio natural es un valor social de inestimable coste asociado al concepto de calidad de vida. Por ello, al valorar la oferta turística del territorio ya no se puede seguir sosteniendo el desarrollo turístico en los términos de los años sesenta. Un proceso de cambio que tiene su culminación en la acuñación del concepto de turismo sostenible al considerarlo soportablemente ecológico, viable económicamente y equitativo socialmente.

Desde principios de los años ochenta se produce un desplazamiento en el interés del turista europeo y nacional por el medio natural y su valor social como por el concepto de calidad de vida. Aquellas valoraciones y situación económica favorable impulsaron el cambio de una nueva perspectiva que conduce a la formulación del concepto de desarrollo sostenible acuñado desde 1987 en el informe titulado «Nuestro Futuro Común» elaborado por la Comisión Brundtland. La OMT (Organización Mundial del Turismo), a partir de este informe, define el desarrollo turístico sostenible como el que «atiende a las necesidades de los turistas actuales y de las regiones receptoras y al mismo tiempo protege y fomenta las oportunidades para el futuro». Se concibe como la gestión de todos los recursos de forma que se pueden satisfacer todas las necesidades económicas, sociales y medioambientales.

No obstante, el carácter generalizado de la definición comporta no saber con exactitud que es una actuación sostenible en cada situación concreta ya que cada agente social puede entender la aceptación del término como quiera a pesar de la evidencia del contenido 
conceptual. Esta libertad interpretativa del paradigma del desarrollo sostenible comporta que el concepto sea objeto de una serie de ideas pervertidas que limitan su consideración general. La primera procede del mundo de los negocios al convertir el desarrollo sostenible en sinónimo de crecimiento sostenible. También, la perversión del concepto por la de utilización sostenible de una corriente que promociona el llamado uso racional cuando su práctica es contraria. Nivel de perversión que se acrecienta cuando el desarrollo sostenible se asimila al de consumo sostenible. Por ello, se hace necesario replantear el modelo de desarrollo sostenible sobre otras premisas como: reparto más equitativo de los recursos, reorientación de los flujos de intercambios e integración de los elementos económicos, sociales y ambientales en el desarrollo de los lugares.

Sin embargo, reconocidos y admitidos los tres ejes básicos del desarrollo sostenible: crecimiento económico, sostenibilidad ambiental y equidad social, la sostenibilidad se concibe como un proceso de cambio cualitativo para alcanzar los objetivos. Por ello, el documento que desarrolla los fundamentos conceptuales y metodológicos del proyecto de investigación: Planificación y gestión sostenible del turismo ${ }^{2}$ propone una definición del desarrollo sostenible útil y operativa para la planificación y gestión de la actividad turística. Esta identifica «el desarrollo turístico sostenible con un proceso de cambio cualitativo producto de la voluntad política que, con la participación imprescindible de la población local, adapta el marco institucional y legal así como los instrumentos de planificación y gestión, a un desarrollo turístico basado en el equilibrio entre la preservación del patrimonio natural y cultural, la viabilidad económica del turismo y la equidad social del desarrollo».

El traslado de esta nuevas propuesta y su aplicación al desarrollo turístico-residencial de la comarca debe pasar por el esfuerzo de analizar y comprender las totalidades complejas del conjunto de elementos interrelacionados que permitan alcanzar una solución definitiva y adecuada. Esta pasa por hacer compatible la mejora de la condición socioeconómica con el mantenimiento del medio ambiente. La forma preferible de evaluar la situación socioeconómica y medioambiental del recurso agua en el espacio litoral y su área de influencia es mediante una serie de indicadores que sinteticen su tendencia sobre las condiciones y los fenómenos ambientales relacionados con su cantidad y calidad.

\subsection{Sistema de indicadores medioambientales y valoración del desarrollo sostenible. El modelo referencial del espacio litoral y su zona de influencia}

Los indicadores son magnitudes de síntesis que facilitan la interpretación de los resultados obtenidos de la actividad humana. Su significado supera el efecto de su propia estadística aportando información que permite tener una medida eficaz de la presión que las acciones humanas ejercen sobre el medio ambiente y la respuesta efectiva proporcionada por las políticas ambientales. Trasladados al campo de los recursos de hídricos reflejan sintéticamente la situación socioeconómica y medioambiental de la gestión del agua (cantidad, racionalización, ahorro, depuración, reutilización etc.). El desarrollo de estos parámetros se ha dirigido hacia objetivos ambientales para alcanzar un considerable nivel de desarrollo sostenible: garantizar el aprovechamiento de los recursos, proteger la salud humana y el bienestar general de la población y conservar la integridad de los ecosistemas.

2 VERA, J. F., IVARS, J. A., JUÁREZ, C. et al., (2001): Planificación y gestión del desarrollo turístico sostenible: propuesta para la creación de un sistema de indicadores. Documentos de Trabajo № 1 . Instituto Universitario de Geografía. Universidad de Alicante, pp. 9. 
Su aplicación les convierte en instrumentos útiles para realizar análisis comparativos entre los diferentes municipios, comarcas y regiones al integrar aspectos medio ambientales en las políticas socioeconómicas y sectoriales.

La OCDE ha aplicado a su sistema de indicadores el modelo Presión-Estado-Respuesta. Este paradigma refleja las relaciones entre las presiones del hombre sobre el medio, los cambios que provocan y las respuestas políticas diseñadas para equilibrar las actividades humanas y mantener las condiciones medioambientales. Y es que, las acciones transformadoras realizadas por los grupos humanos ejercen presión sobre el territorio y cambian la calidad y la cantidad de los recursos de agua. La sociedad responde a estas alteraciones mediante políticas ambientales, sectoriales y económicas (respuestas sociales). Proceso que genera una interacción constante entre la presión sobre el medio y la respuesta ofrecida por los agentes sociales. En consecuencia, el modelo se organiza con base a los tres tipos de indicadores citados que incluyen la percepción del problema, la formulación de las políticas y el seguimiento y evaluación de las mismas.

Los indicadores económicos y sociales se conforman al final de la década de los cincuenta y se consolidan en los años sesenta con el objetivo de diferenciar el grado de desarrollo entre regiones y países. Estos han sido el origen de los indicadores ambientales pero el concepto y el tiempo de aplicación es diferente porque la teoría general del desarrollo económico sostenido tiene sus fundamentos en otros principios y criterios relacionados con el agotamiento de los recursos naturales y la degradación ambiental y el posible freno al desarrollo ${ }^{3}$.

Los indicadores de Presión describen las presiones directas e indirectas ejercidas sobre el medio ambiente por las actividades humanas, como es el caso de la creciente demanda por el aumento de población y paralelamente el aumento de aguas residuales generadas.

Los indicadores de Estado-Calidad se refieren a la calidad del medio ambiente en un momento determinado como a la cantidad y estado de los recursos naturales, por ejemplo, la cantidad de agua embalsada de posible utilización.

Los indicadores de respuestas se corresponden con los esfuerzos realizados por la sociedad para atenuar o reducir la degradación del medio natural. La mejor forma de evaluar esta acción es mediante las inversiones crecientes realizadas por las empresas o municipios en el tratamiento de las aguas residuales.

El agua es un recurso escaso, insustituible, vulnerable, susceptible de usos alternativos y elemento importante como receptor de vertidos. Es indispensable para la actividad humana y está sujeta a un ciclo permanente en el que la intervención del desarrollo económico está modificando sus características. Esta situación requiere, cada vez con mayor frecuencia, conocer las existencias y su calidad con el objetivo de aumentar el volumen utilizable, proteger su importancia y ahorrar su uso en armonía con el resto de los recursos naturales. Cuando uno de estos recursos es imprescindible y de problemática disponibilidad la armonización suscita tensiones difíciles de resolver por su escasez y grado de dependencia.

Los principales problemas ambientales relacionados con el agua en los municipios turísticos de la comarca de la Vega Baja son: la escasez del recurso para satisfacer las distintas demandas crecientes y el deterioro de la calidad. La masificación turística tiene una serie de efectos negativos sobre el medio: aumento del consumo de suelo, agua y energía. Para considerar a un territorio como dominio turístico sostenible es necesario

3 MINISTERIO DE MEDIO AMBIENTE (2000). Indicadores Ambientales. Una propuesta para España. Madrid, pp. 9. 
alcanzar un nivel considerable de reducción, reutilización y reciclado de los residuos como una buena gestión del agua potable y residuales. Se trata, en el caso de los recursos hídricos, de conocer la situación actual (diagnóstico), satisfacer las demandas futuras generadas por el modelo de desarrollo económico (objetivo) y enumerar las propuestas para aumentar las disponibilidades que garanticen un desarrollo económico prolongado en el tiempo. Proceso de la estrategia de la sostenibilidad en el mundo turístico, desde el campo de los recursos hídricos y su gestión eficiente, conformado sobre la protección de la calidad y el control de las aguas residuales.

\subsubsection{Indicadores medioambientales de Estado-Calidad}

El desarrollo sostenible comporta crecimiento económico y calidad de vida en equilibrio con los recursos de agua. El turismo con su correspondiente ocio y el consumo urbano han aumentado la presión sobre los recursos hídricos del territorio. El compromiso de sustituir el modelo tradicional de desarrollo turístico por el de naturaleza sostenible debe tener en cuenta, desde el campo de los recursos hídricos y su gestión eficiente, los objetivos de minimizar el consumo de agua y maximizar la calidad del producto. Como las grandes transformaciones territoriales del desarrollo local se han fundamentado en el mayor uso del agua para cualquiera de sus actividades productivas se han realizado previamente los balances hidráulicos de su ámbito de aplicación.

Los indicadores hídricos de sostenibilidad aceptados por la comunidad científica se acomodan a escala de país como a la unidad comarcal. Su realización permite comprobar en el ámbito de la Vega Baja la existencia de un elevado déficit (relación entre volúmenes disponibles y uso), la escasa disponibilidad de recursos de agua por la cantidad de precipitación anual recibida frente a la elevada ETP (evapotranspiración potencial o necesidad de agua) como la presencia de un acuífero de mala calidad del agua para abastecimiento, elevado grado de salinidad y recarga dominante procedente de la infiltración de retorno de riegos (Cuadro $\mathrm{n}^{\mathrm{o}} 1 \mathrm{y} \mathrm{n}^{\mathrm{o}} 2$ ).

Estas características del balance hídrico comarcal no guardan relación con el correspondiente de otras unidades hidrográficas, caso de la cabecera del río Tajo. En este territorio las condiciones de lluvia y su efectividad confirman una aportación media anual ligeramente superior a los $1.400 \mathrm{hm}^{3}$ /año que convenientemente regulados por los embalses del Estado (Entrepeñas y Buendía con capacidad conjunta de $2.442 \mathrm{hm}^{3}$ ) pueden servir para atender las necesidades requeridas en la cuenca del Segura sin condicionar el desarrollo económico de la cuenca del Tajo ${ }^{4}$. Indicador (balance hídrico) que permite poner de manifiesto las diferencias internas del territorio español encubiertas por la realización del balance a escala nacional.

La realización del balance hídrico comarcal confirma la escasa disponibilidad de recursos de agua para el desarrollo agrario como para la implantación de industrias y el crecimiento turístico-residencial. El indicador de disponibilidad considera recursos disponibles por habitantes, los procedentes de la regulación del agua superficial, de los caudales fluyentes garantizados en régimen natural y de las extracciones de acuíferos en situación de sobreexplotación. Las condiciones descritas en el balance hídrico confirman a los municipios turísticos como un área de escasa disponibilidad de recursos por habitante para abordar cualquier acción de mejora socioeconómica de garantizada rentabilidad. No se

4 JUÁREZ SÁNCHEZ-RUBIO, C. (1991): Planificación hidrológica y desarrollo económico: El trasvase Tajo-Segura. Instituto de Cultura «Juan Gil-Albert». Alicant, pp. 19. 


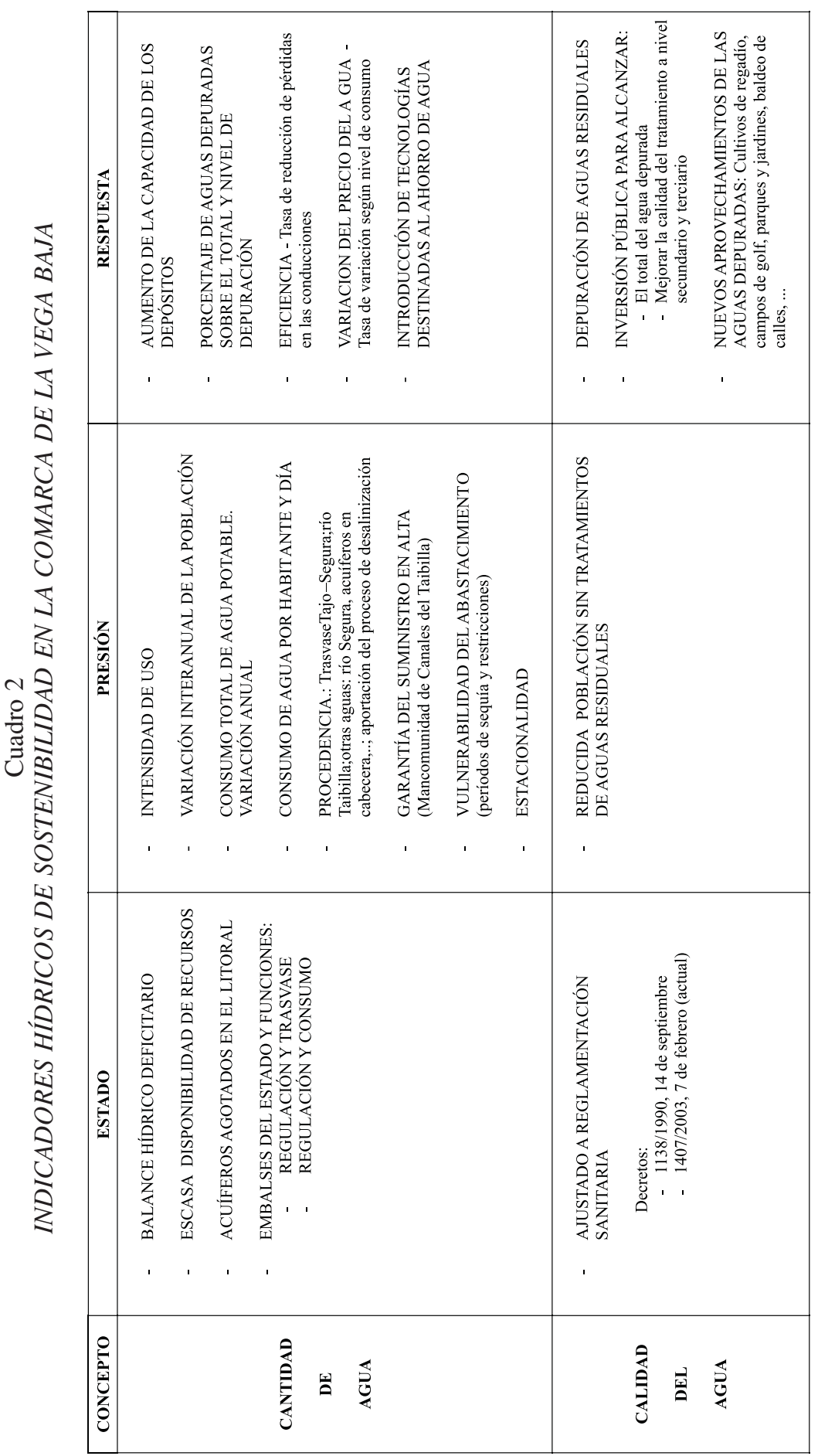


dispone de caudales superficiales regulados como de caudales fluyentes naturales porque el suelo almacena el escaso volumen del período más favorable del balance hídrico, no produciéndose tiempo alguno de exceso de agua de posible regulación.

La comarca acoge un acuífero constituido por materiales arenosos y calizos de poco espesor con recursos escasos y calidad del agua mala o regular. Abarca una extensión de $750 \mathrm{~km}^{2}$ que debido a la heterogeneidad de los materiales hace difícil evaluar las reservas de agua almacenada durante tiempos geológicos aunque han sido calculadas en $500 \mathrm{hm}^{3}$ hasta una profundidad de 100 metros $^{5}$. El mismo estudio estima que la recarga media anual es de $75 \mathrm{hm}^{3} /$ año procedente de la lluvia, infiltración de retornos de riegos, pérdidas en las redes de las acequias, y alimentación subterránea lateral. La calidad de sus aguas varía considerablemente entre un mínimo de 5 gr/litro y un máximo de 15 gr/litro. Condiciones potenciales que debidamente explotadas mediante el proceso de desalinización se convierten en una alternativa valiosa para uso agrícola pero no como el medio de solucionar el déficit hídrico. Por ello, la explotación anual se cifra en $50 \mathrm{hm}^{3}$ no considerando al acuífero como sobreexplotado ya que el volumen de agua extraída no se realiza de las reservas no renovables de la unidad hidrológica.

Los indicadores medioambientales de Estado-Calidad transportados al sector marítimo y su área de influencia confirman su debilidad ante la necesidad de conformar un plan estratégico de desarrollo sostenible. La cantidad de agua como su garantía en el abastecimiento es el mayor factor de debilidad económica del territorio. También su calidad. Un concepto relativo a un uso por lo que las exigencias dependerán del empleo a que esté destinado el recurso. Cuando su utilización es para suministro urbano el distribuidor en alta tiene necesidad de ajustarse al marco legal impuesto por la reglamentación sanitaria.

En este cometido la Mancomunidad de los Canales del Taibilla empresa gestora de los caudales transferidos desde le río Tajo ajustaba la calidad del agua consumida a lo dispuesto en el Real Decreto 1138/1990, de 14 de agosto. Esta situación ha variado por la exigencia de incorporar las directrices de la Directiva 98/83/C, de 3 de noviembre de 1998. El control de la calidad del agua de consumo humano exigido por la reglamentación técnico sanitaria se establece mediante el Real Decreto 140/2003, de 7 de febrero. Un marco legal más acorde, tanto con las necesidades actuales como con los avances y progresos, que establece las medidas sanitarias y de control para la protección de la salud de los consumidores en el nivel local. La MCT verifica en los laboratorios de sus Estaciones de Tratamiento, los ensayos físico-químicos y bacteriológicos fijados por la reglamentación vigente. Se considera, por tanto, que la calidad del agua está ajustada a la Reglamentación y es disponible para su consumo.

Los indicadores medioambientales de Estado-Calidad ponen de manifiesto la gran desigualdad de distribución de los recursos de agua y la posibilidad de solucionar el desequilibrio hidráulico mediante la incorporación de los procesos técnicos sin alterar las condiciones medioambientales. Modificar las pautas de la naturaleza para trasladar artificialmente a los lugares donde falta agua el exceso de otros territorios es factible y debe continuar siempre que estén justificados los aspectos técnicos, ecológicos y económicos.

\subsubsection{Indicadores hídricos de Presión}

El modelo turístico de sol y playa tiene sus consecuencias más negativas en el permanente crecimiento económico y en el número de visitantes, superando la capacidad de

5 MEDINA, A. et al. (1992): Las agua subterráneas en la provincia de Alicante. Diputación de Alicante e Instituto Geológico y Minero de España (Mecanografiado). 
acogida del territorio. Este modelo no se ha agotado. La confirmación se realiza mediante el aumento de turistas que llegan de diferentes lugares. La afluencia masiva de visitantes a la zona turística-costera crece en forma residencial como estacional. La población extranjera es la principal causa de este incremento relacionado con el clima y la presencia de campos de golf del sector litoral. Este factor positivo está provocando un crecimiento urbanizador por la adquisición de viviendas que contribuye a superar la estacionalidad. Mientras el modelo alternativo, turismo sostenible, tiene escasa repercusión práctica.

El debate se centra en el cambio de modelo. Este no parece adecuado para lograr un desarrollo sostenido. Se trata de encontrar otro que a menor coste ofrezca una rentabilidad económica, medio ambiental y social similar o superior. Se quiere optar por un turismo de clase media-alta más minoritario que consume menos recursos y eleva la rentabilidad como Baleares y Canarias. Los municipios litorales y su área interior de la comarca se Cuadro 3

INDICADORES DE PRESIÓN DEL AGUA EN LOS MUNICIPIOS TURÍSTICOS DEL BAJO SEGURA

\begin{tabular}{|c|c|c|c|c|c|c|}
\hline \multirow{2}{*}{ MUNICIPIOS } & \multirow{2}{*}{$\begin{array}{l}\text { Consumo } \\
\mathrm{m}^{3} / \mathrm{año}\end{array}$} & \multirow{2}{*}{ Procedencia (1) } & \multirow{2}{*}{$\begin{array}{c}\text { Índice de } \\
\text { estacionalidad }\end{array}$} & \multirow{2}{*}{$\begin{array}{c}\text { Porcentaje } \\
\text { de } \\
\text { aumento } \\
\text { población } \\
(1991- \\
2001) \\
\end{array}$} & \multicolumn{2}{|c|}{$\begin{array}{c}\text { Consumo } \\
\text { (en } \\
\text { litros/hab/día) }\end{array}$} \\
\hline & & & & & $\begin{array}{l}\text { Pob P } \\
\text { (2) }\end{array}$ & $\begin{array}{l}\text { Pob E } \\
\text { (3) }\end{array}$ \\
\hline $\begin{array}{c}\text { Guardamar del } \\
\text { Segura }\end{array}$ & 1.760 .867 & Diversificada & $\mathrm{A} / \mathrm{F}=3,48$ & $66 \%$ & 170 & 220 \\
\hline Orihuela & 8.024 .048 & Diversificada & $\mathrm{A} / \mathrm{E}=2,18$ & $9.6 \%$ & 250 & 350 \\
\hline $\begin{array}{l}\text { Pilar de la } \\
\text { Horadada }\end{array}$ & 2.476 .562 & Diversificada & $\mathrm{A} / \mathrm{F}=2,61$ & $66.7 \%$ & 250 & 350 \\
\hline Rojales & 1.380 .856 & Diversificada & $\mathrm{JL} / \mathrm{E}=2,18$ & $62.4 \%$ & 120 & 250 \\
\hline San Fulgencio & 714.338 & Diversificada & $\mathrm{A} / \mathrm{E}=2,28$ & $53.4 \%$ & 175 & 220 \\
\hline $\begin{array}{c}\text { San Miguel } \\
\text { de Salinas }\end{array}$ & 495.970 & Diversificada & $\mathrm{JL} / \mathrm{E}=1,70$ & $50.3 \%$ & 150 & 220 \\
\hline Torrevieja & 8.813 .524 & Diversificada & $\mathrm{A} / \mathrm{E}=3,10$ & $96.8 \%$ & 140 & 350 \\
\hline TOTAL & 23.666 .165 & & & $39.7 \%$ & & \\
\hline
\end{tabular}

(1) Procedencia del agua: Diversificada (Trasvase Tajo-Segura), río Taibilla, desalación y otras aguas

(2) Población permanente

(3) Población estacional 
enfrentan a varios problemas si mantienen el modelo de atraer un turismo masivo. El primero proviene de la limitación de los recursos de suelo y agua. La dificultad en el suministro de agua es grande. El vector demográfico se establece como la causa de los desajustes económicos, sociales y ambientales.

El crecimiento demográfico ejerce una creciente presión sobre los recursos limitados y deteriora la calidad de los recursos existentes. Entre el año 1960 y el 2001 la población aumenta en la comarca en un $73 \%$. También se produce un crecimiento importante en otros territorios próximos que se abastecen en alta de la misma empresa gestora, la MCT. La entidad avanza la previsión de que en el año 2020 el aumento demográfico y el turismo triplicarán el consumo de agua en el litoral de las provincias de Alicante y Murcia. Aumento urbanístico y consiguiente incremento de la población que no llevan parejo la puesta en marcha de otros servicios que producen importante impacto ambiental. La planificación tiene que apuntar a equilibrar los recursos disponibles, a proteger y mejorar la calidad mediante el uso de las mejoras técnicas, la participación de la población, las estructuras económicas y financieras y la fijación de metas reales.

El desarrollo económico sostenible busca influenciar, alentar y ayudar a los municipios a conservar la integridad y la diversidad de la naturaleza como asegurar que todo uso de los recursos naturales sea equitativo y ecológicamente sustentable. Es la manera más responsable de realizar una planificación y gestión sostenible. El proceso de la estrategia de la sostenibilidad en el territorio turístico de la Vega Baja debe conformarse sobre el control de los recursos de agua potable y su utilización de manera eficiente y equitativa como del control de las aguas residuales. Y es que, el desarrollo turístico-residencial del sector litoral, de considerable rentabilidad pero no sostenible por la escasez de agua, basa su estrategia en la utilización exagerada de la construcción y la dependencia externa del agua. Modelo de desarrollo contrario al concepto de desarrollo sostenible por la dependencia exagerada que se establece sobre el sistema productivo como por su propensión a la degradación.

Los indicadores de presión informan de las comprensiones directa e indirecta sobre el medio. La OCDE utiliza a la intensidad de uso del agua como un parámetro significativo por la advertencia que transmite. Sin embargo, su definición: «considerada como el cociente entre las extracciones primarias netas de agua y la escorrentía total resultante de restar a la precipitación la evapotranspiración potencial» carece de interés práctico y de muy difícil realización por lo que ha sido cuestionado y suprimido en el elenco de los indicadores en el proceso de valoración. En cambio, consideramos que otra serie de variable son más útiles para explicar la presión sobre los recursos como: variación de la población, aumento del consumo total de agua, garantía de suministro, estacionalidad, etc. (Cuadro $\mathrm{n}^{\mathrm{o}} 3$ ).

La población censada aumenta considerablemente y su valor absoluto y relativo ha sido indicado anteriormente. En estrecha relación con el crecimiento demográfico y la mejora económica se ha producido un aumento del consumo de agua en los municipios turísticos y en el resto del espacio comarcal como en el área de abastecimiento de la MCT. El proceso tiene su mayor repercusión en los municipios costeros de Guardamar del Segura, Orihuela, Pilar de la Horadada y Torrevieja donde el volumen de agua consumido anualmente ha variado de $5,49 \mathrm{hm}^{3}(100 \%)$ en 1979 , antes de la realización del trasvase TajoSegura, a $13,05 \mathrm{hm}^{3}$ en el año 1991 y a $21,09 \mathrm{hm}^{3}$ en el año 2000, que en término relativo se eleva al $384 \%$. Es decir, en algo más de dos décadas el consumo de agua de los cuatro municipios del litoral casi llega a multiplicarse por cuatro. Aumento del consumo de agua y grado de concentración que se confirma cuando se incluyen en el análisis los municipios de la segunda línea como Rojales, San Fulgencio y San Miguel de Salinas pasando de $14,93 \mathrm{hm}^{3}$ en 1991, el $47 \%$ de toda la comarca a 23,66 hm³ , el 74,5\%, en el año 2000 (fig. 
$\mathrm{n}^{\mathrm{o}}$ 2). El territorio turístico residencial se individualiza como un espacio de crecimiento demográfico y de concentración de recursos de agua en el desigual proceso de desarrollo económico-social de la comarca.

No obstante, este modelo turístico presenta grados de intensidad que varían desde el nivel máximo de Torrevieja hacia los municipios de segunda línea y los de reciente incorporación. En Torrevieja se han construido algo más de 78.000 viviendas, mayoritariamente destinadas al turismo de segunda residencia, en el corto período de tres lustros, 1985-2000, con previsiones de construcción de 38.000 viviendas más hasta el año 2008. Un crecimiento urbanístico sin precedente en España que lleva aparejado un aumento demográfico confirmado estadísticamente en la variación del número de personas censadas en $1981,12.314(100 \%)$ y las $50.953(414 \%)$ en el año 2001. Este porcentaje guarda una estrecha relación con el volumen de agua consumida al pasar de 2,05 hm $\mathrm{hm}^{3}(100 \%)$ en 1981 a $8,81 \mathrm{hm}^{3}$ en el año 2000 (430\%). Evolución de caudales aportados, registrados y número de abonados al servicio de agua potable entre los años 1980 y 2000 (fig. no 3) que confirman el desarrollo urbanístico y el crecimiento demográfico.

Estos valores alcanzados singularizan al municipio como el de mayor crecimiento relativo de todo el país y su tasa de expansión urbanística se corresponde con un aumento del consumo de agua que tiene en el número de abonados al servicio municipal de agua potable un incremento de casi el $7 \%$ en el año 2000, hasta alcanzar la cifra de 90.500 , frente a los 84.400 de principio de año (fig. $n^{\circ} 3$ ). Proceso que tiene su repercusión en los servicios de estacionalidad, dimensionamiento de las infraestructuras con base a previsiones de crecimiento, infraestructuras en continua ampliación y un servicio integral del ciclo del agua sometido al diferente grado de ocupación temporal en las urbanizaciones.

Figura 2. Abastecimiento de agua a Municipios Turísticos del Bajo Segura 1991-2000

Fuente: Mancomuidad de Canales del Taibilla. Elaboración propia

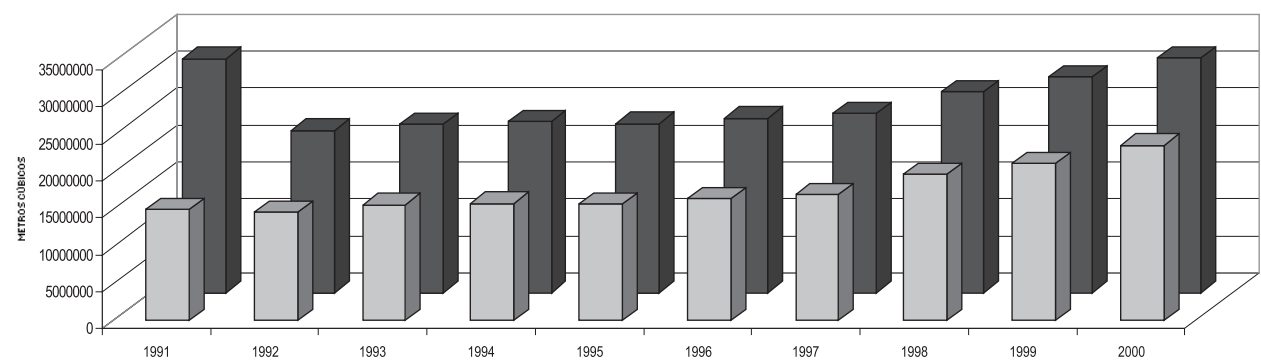

El suministro de agua a los municipios de la comarca es mediante la Mancomunidad de los Canales del Taibilla organismo autónomo cuya funcionalidad es estrictamente gestora de los recursos desde los principios que le determina la Dirección de Obras Hidráulicas, a la que verdaderamente corresponde decidir la admisión de nuevos municipios. La eficacia de la MCT en la distribución junto a la gestión que llevan a cabo las empresas concesionarias y la administración municipal de la distribución de agua potable en baja garantizan el suministro durante todo el año. Garantía que rompe la estacionalidad y se convierte en el elemento creador de una nueva actividad productiva de mayor rentabilidad por unidad de agua utilizada. 
Junto al indicador de presión sobre el medio, referido al consumo total de agua potable y su variación anual como el gasto por habitante y día, se toma en consideración la procedencia del agua aportada. El 70\% del agua gestionada por la MCT durante el período 1981-2000 procede de la cabecera del río Tajo. La Ley 52/80, de 16 de octubre fija el destino de $110 \mathrm{hm}^{3} /$ año para el abastecimiento de los municipios consorciados. Este volumen ha crecido hasta $131 \mathrm{hm}^{3} /$ año por la confluencia de la reducción de pérdida en el transporte y la flexibilidad en la distribución. Aportación anual foránea que se acrecienta con las aguas del río Taibilla y otras contribuciones. El desarrollo urbanístico, demográfico y nivel de vida frente a los recursos limitados plantea un gran dilema. Es necesario saber no sólo de donde va a salir el agua cuando se plantean nuevas construcciones urbanísticas sino el modelo de gestión del agua fundamentado en la oferta continuada o en el alternativo de gestión en la demanda.

El modelo desarrollista de los años sesenta fundamenta su crecimiento económico en la oferta continuada de agua y en la transferencia a los territorios donde se podía alcanzar el mayor beneficio económico y de generación de empleo. Este modelo parecía superado. El crecimiento ilimitado de los recursos limitados es completamente imposible. Es necesario poner límite a este crecimiento en desarmonía con los recursos de agua. Sin embargo, para no limitar el crecimiento urbanístico se aumenta la disponibilidad del recurso mediante el proceso técnico de la desalinización como solución puntual a los problemas estructurales de necesidad de agua, mientras llega el trasvase del Ebro. Mejora técnica que genera caudales considerables de agua pero provocan impacto en el medio ambiente por el consumo de energía y las dificultades derivadas de la concentración de sal procedente del proceso de ósmosis inversa.

La MCT dispone de una planta desaladora en el término municipal de Alicante que ha entrado en funcionamiento para aportar $18 \mathrm{hm}^{3} /$ anuales y otra en proyecto con la misma producción anual de $18 \mathrm{hm}^{3} /$ año. La nueva infraestructura hidráulica permite liberar un volumen equivalente que se reparte entre el resto de localidades dependientes de la entidad abastecedora. Además, la nueva desaladora forma parte de un programa de ejecución de cuatro instalaciones localizadas en la región de Murcia (San Pedro del Pinatar con capacidad para producir $24 \mathrm{hm}^{3} /$ año y otra en el Campo de Cartagena con $\left.57 \mathrm{hm}^{3} / \mathrm{año}\right)^{6}$. En un período corto de tiempo la disponibilidad de caudales para abastecimiento se puede ver incrementado entre 80 y $100 \mathrm{hm}^{3} /$ año. Nueva oferta de agua que implica volver a las tasa de crecimiento anual de viviendas de finales de los años ochenta. Dinámica que no guarda relación alguna con la coherencia del discurso asumido sobre el desarrollo sostenible y el modelo de gestión sostenible del agua en el marco territorial del sureste peninsular. Por ello, cualquier actuación urbanística que se quiera realizar debería obligatoriamente contar con el recurso agua. La mejor forma de cumplir el criterio de sostenibilidad.

La presión y las obras realizada para disponer de mayor volumen de agua y garantizar el abastecimiento de la población, rompiendo la estacionalidad, no han sido capaces de asegurar todas las demandas generadas. El abastecimiento tiene que soportar la presión que conllevan los períodos de sequía provocando restricciones y vulnerabilidad en el suministro urbano. El elevado grado de dependencia del abastecimiento de los centros urbanos turístico de los caudales proporcionados por la cabecera del río Tajo implica que los períodos de sequía de esta cuenca hidrográfica tengan su repercusión en la disminución del caudal transferido. La sequía es un fenómeno climático con el cual hay que contar y tratar de vencer con todos los medios posibles. Considerada como un mínimo hidrológico respecto al valor normal, es un desequilibrio pluviométrico difícil de predecir y de poca garantía su final.

6 MINISTERIO DE MEDIO AMBIENTE (2003): Revista Ambiente, no 27, pp. 18-22. 
La irregularidad de las precipitaciones es una característica del clima de la cabecera del río Tajo. La diferencia entre el año más lluvioso y el más seco arroja un valor de 500 milímetros, equivalente a 500 litros por metro cuadrado. El coeficiente de variación de irregularidad es de la magnitud de 4,5 (relación entre aporte máximo, $1.800 \mathrm{hm}^{3} \mathrm{y}$ el aporte mínimo de $400 \mathrm{hm}^{3}$ ) y su recorrido de $1.400 \mathrm{hm}^{3}$. Estas condiciones junto con las de la cuenca del Segura (en el año hidrológico 1994-95 se recibieron sólo $135 \mathrm{hm}^{3}$ ) ponen de manifiesto cierto grado de correlación positiva entre ambas. Es decir, en términos generales, tanto los períodos de abundancia como los de sequía se producen conjunta y simultáneamente en sus territorios. Esta coincidencia explica la vulnerabilidad del abastecimiento que comporta presión sobre los acuíferos y restricciones

La estacionalidad (relación entre el consumo del mes máximo y mínimo) es un nuevo indicador mediante el cual se ofrece información concisa y representativa de las actuaciones e infraestructuras necesarias para lograr la satisfacción de la demanda. La estacionalidad es el principal problema que afecta a la rentabilidad de la oferta turística provocando desigualdad en el uso. Lo más significativo es que en los cuatro municipios marítimos este valor supera el nivel dos (2) para alcanzar los mayores índices en los tradicionales centros turísticos como Torrevieja y Guardamar del Segura con valores superiores tres (3), (Cuadro $\mathrm{n}^{\mathrm{o}} 2$ ). Estos índices están estrechamente imbricados con los porcentajes de consumo de agua acaparado por los meses de verano (julio, agosto y septiembre), 40 y $38 \%$, respectivamente, mientras en el resto de los municipios turísticos el porcentaje varía del $30 \%$ en San Miguel de Salinas al 35\% en Pilar de la Horadada.

Sin embargo, los valores del índice de estacionalidad están disminuyendo significativamente. Se está produciendo en la zona un importante incremento de la desestacionalidad del sector turístico desde mediados de la década de los años noventa. En Guardamar del Segura se pasa de 3,85 en 1991, a 3,48 en el año 2000; en Pilar de la Horadada(independizada de Orihuela desde 1986) se pasa de 3,55 a 2,28 y en Torrevieja de 3,37 a 3,10. Solamente en Orihuela cambia la tendencia al pasar de 1,34 a 2,18 porque desde 1998 el Ayuntamiento suministra agua a urbanizaciones que hasta ahora se abastecían mediante pozos que han terminado por salinizarse (Cuadro $\mathrm{n}^{\mathrm{o}} 3$ ). Indicador expresivo del grado de intensidad del proceso turístico, concentración temporal y espacial y propensión de cambio como explicativo del aumento de la población residente, estacional y flotante.

Esta nueva tendencia del índice de estacionalidad como las campañas llevadas a cabo para concienciar el uso racional del agua potable tienen su manifestación estadística expresa en el consumo de litros por habitante y los contrastes internos no sólo entre sus municipios sino entre la población permanente y estacional. No obstante, la mayor concentración de población en la zona turística-residencial se produce en los quince primeros días del mes de agosto pudiéndose alcanzar la cantidad de 740.000 personas con máximo en Torrevieja de 420.800 y de 135.000 en Orihuela. La confluencia en el término municipal de Torrevieja del máximo de habitantes con mayor consumo estacional determina la mayor demanda del año que llega hasta los 50.000 metros cúbicos. Volumen de agua consumido semejante al proporcionado diariamente por la Desalinizadora del Canal de Alicante inyectado a la red de la Mancomunidad de los Canales del Taibilla.

\subsubsection{Indicadores hídricos de respuesta}

La afluencia masiva de visitantes y residentes en los municipios turísticos ha modificado su condición económica y generado empleo por la garantía del abastecimiento urbano. Sin embargo, las leyes que rigen la administración del suelo quieren liberalizar más la disponibilidad del territorio y acentuar la saturación que ya se soporta en varios munici- 
pios. Como el planeamiento empresarial está regido por la maximización del beneficio se puede llegar a producir una sobreoferta de viviendas residenciales que pueden llegar a devaluar el entorno paisajístico. Los indicadores de presión han puesto de manifiesto que el modelo turístico de crecimiento continuado debe ser sustituido por otro más en consonancia con la competitividad económica, la justicia social y el respeto al medioambiente. Por ello, los gobernantes tienen la obligación de resolver el problema con el menor costo económico, social y ambiental posible.

Los indicadores de respuesta tienen el objetivo de comprobar el esfuerzo económico (gastos de inversión) y medioambiental realizado por los grupos humanos para reducir la degradación del litoral, el elevado grado de contaminación que comporta la no depuración de las aguas residuales y reducir la presión sobre el recurso agua.

La necesidad de superar la servidumbre de la estacionalidad para no afectar a la rentabilidad de la oferta turística provoca una fuerte bipolarización de las infraestructuras y equipamiento entre una subutilización invernal y una hipercongestión estival. La confirmación estadística de este proceso queda reflejada en el indicador de la capacidad de los depósitos de los diferentes municipios y las previsiones contempladas por las empresas gestoras o los ayuntamientos. En este sentido, la capacidad de los siete centros urbanos se eleva a casi $100.000 \mathrm{~m}^{3}$ (40\% en Torrevieja), mientras el aumento previsto hacia el futuro y en relación con el planeamiento se estima en $82.700 \mathrm{~m}^{3}$. Una lucha contra la subutilización que se realiza mediante la oferta invernal dirigida a la llegada de jubilados y personas de la tercera edad, lo que potencia aumentar el turismo de residentes establecidos en los diferentes centros urbanos.

Todos los municipios disponen de su correspondiente depuradora aunque no todas las urbanizaciones tienen su alcantarillado conectado con sus correspondientes EDAR (reducida población sin tratamiento de aguas residuales como indicador hídrico de presión). En conjunto, el volumen de agua depurada, $11,07 \mathrm{hm}^{3}$ en el año 2000 , sólo equivale al $47 \%$ del total del agua consumida. Un porcentaje por debajo de las expectativas pero en alza tendente para garantizar la depuración del total de agua utilizada. Se planifica el futuro con el objetivo de recuperar todos los caudales aplicados a cualquiera de los usos contemplados en el territorio. Las directrices trazadas para la total solución se conforman sobre la base de conectar las nuevas y las viejas urbanizaciones con sus respectivas EDAR. La preocupación de las empresas es minimizar las emisiones de aguas residuales a fin de proteger las condiciones medioambientales y sanitarias de la franja litoral (Baño) con incidencia en la principal industria (Turismo).

La respuesta dada por los municipios es sumamente contrastada al variar su eficiencia entre el $60 \%$ y el $100 \%$ del agua recogida en la red (Cuadro no 4 ). Los porcentajes de vertidos no conectados a la red(indicador de presión como población sin tratamiento) son poco significativos: $10 \%$ en San Fulgencio sobre una población de 1.600 habitantes en el censo de 2001, 7\% en Orihuela sobre 49.600 habitantes y 5\% en Rojales y Guardamar del Segura sobre una población censada de 7.500 y 5.300, respectivamente. Alta eficiencia del volumen de aguas depuradas que se confirma con los datos correspondientes a las depuradoras ubicadas en los municipios, tipo de tratamiento e información del destino del efluente (riego, excedentes vertidos al río Segura, campos de golf, parque y jardines). La confirmación estadística del proceso se realiza mediante la variación del volumen de agua depurada en el bienio 2000-01 pasando de los $11,07 \mathrm{hm}^{3}$ del primer año a los $13,89 \mathrm{hm}^{3}$ del segundo. Aumento porcentual medio del $25 \%$ que tiene en el municipio de Orihuela su mayor variación, el $74 \%$, al pasar de $1,59 \mathrm{hm}^{3}$ a $2,72 \mathrm{hm}^{3}$, sin incluir los volúmenes depurados de la zona costera en funcionamiento desde el año 2002 (Cuadro $n^{\circ} 4$ ). 


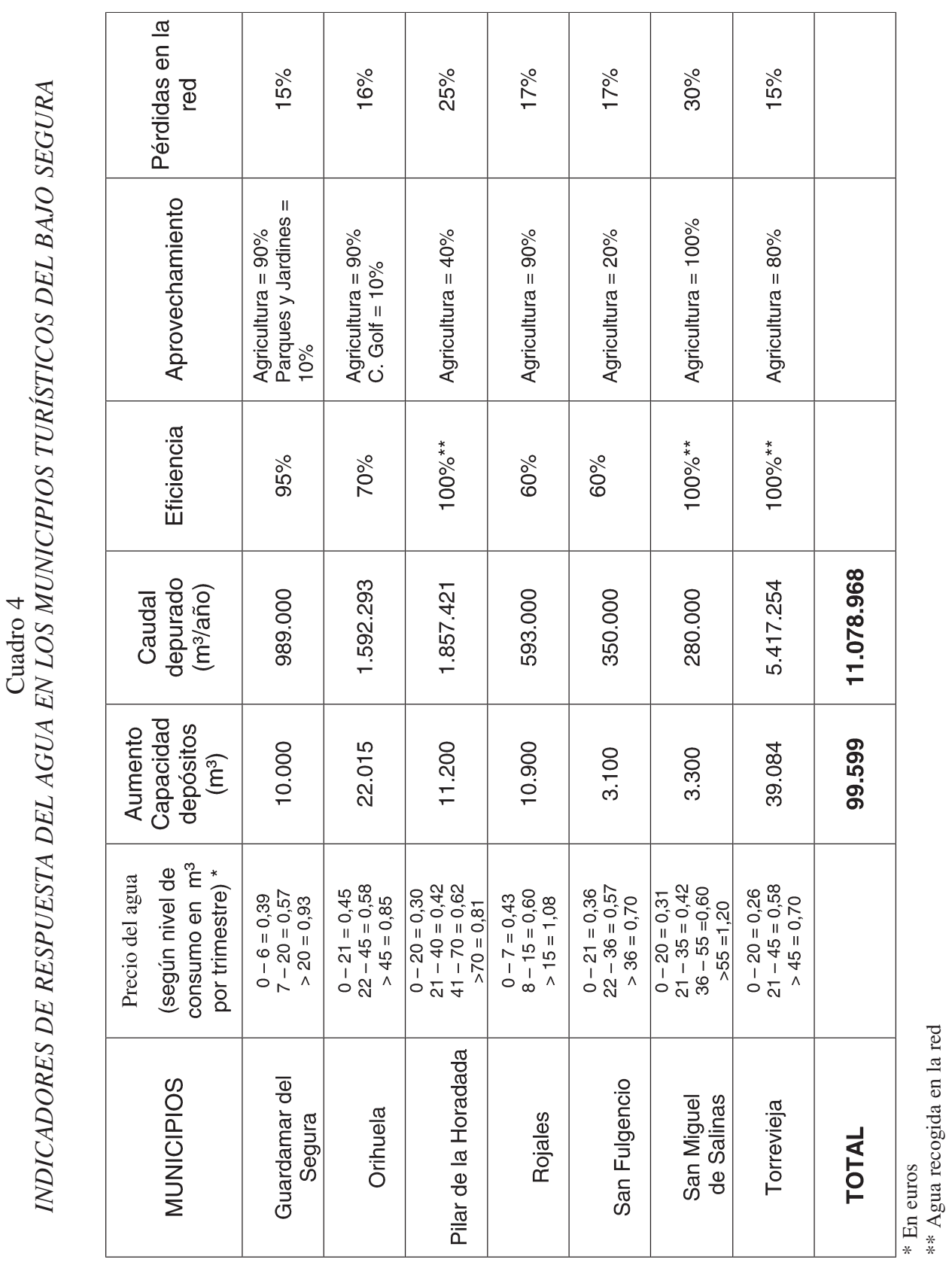


Se configura, por tanto, una línea de acción prioritaria del desarrollo de las infraestructuras hidráulicas de los municipios turísticos para conservar y proteger la calidad de los recursos de aguas residuales generadas por la actividad humana. Estrategia de Desarrollo Sostenible entendida como la conciliación del desarrollo económico y social con la preservación del entorno. Esta actuación sobre el espacio litoral tiene en el municipio de Torrevieja el mayor valedor al depurar $5,4 \mathrm{hm}^{3}$ en el año 2000 de $10 \mathrm{~s} 8,8 \mathrm{hm}^{3}$ introducidos en la red. Volumen de aguas depuradas que se eleva a $6,41 \mathrm{hm}^{3}$ en el año 2001. Un nivel de eficiencia en la depuración del agua utilizada no alcanzado en otras zonas de la provincia. En consecuencia, en aras de un mejor servicio al ciudadano y de conseguir una mejor calidad de las aguas usadas, entendemos que se impone la necesidad de una gestión integrada de todos los servicios de abastecimiento y saneamiento de agua de las poblaciones.

La mejora de la percepción de la opinión pública sobre los problemas ambientales se realiza mediante la aplicación de los indicadores de: eficiencia en su doble sentido de reducción de pérdida de agua en la red como de agua recogida en la misma malla, variación del precio del agua y nuevos aprovechamientos de los caudales depurados. La primera de las acciones tiene su confirmación estadística en la alta optimización de la gestión del abastecimiento urbano de casi todo los municipios ya que las pérdidas se reducen al $15 \%$ en Torrevieja y Guardamar del Segura hasta alcanzar niveles negativos, superiores a la media nacional del 21\%, en Pilar de la Horadada y San Miguel de Salinas con porcentajes del 25 y $30 \%$, respectivamente. Un parámetro que se define como la relación entre el agua suministrada a la red y el agua registrada en los contadores de los abonados (pérdidas) (fig. $n^{\circ} 3$ ).

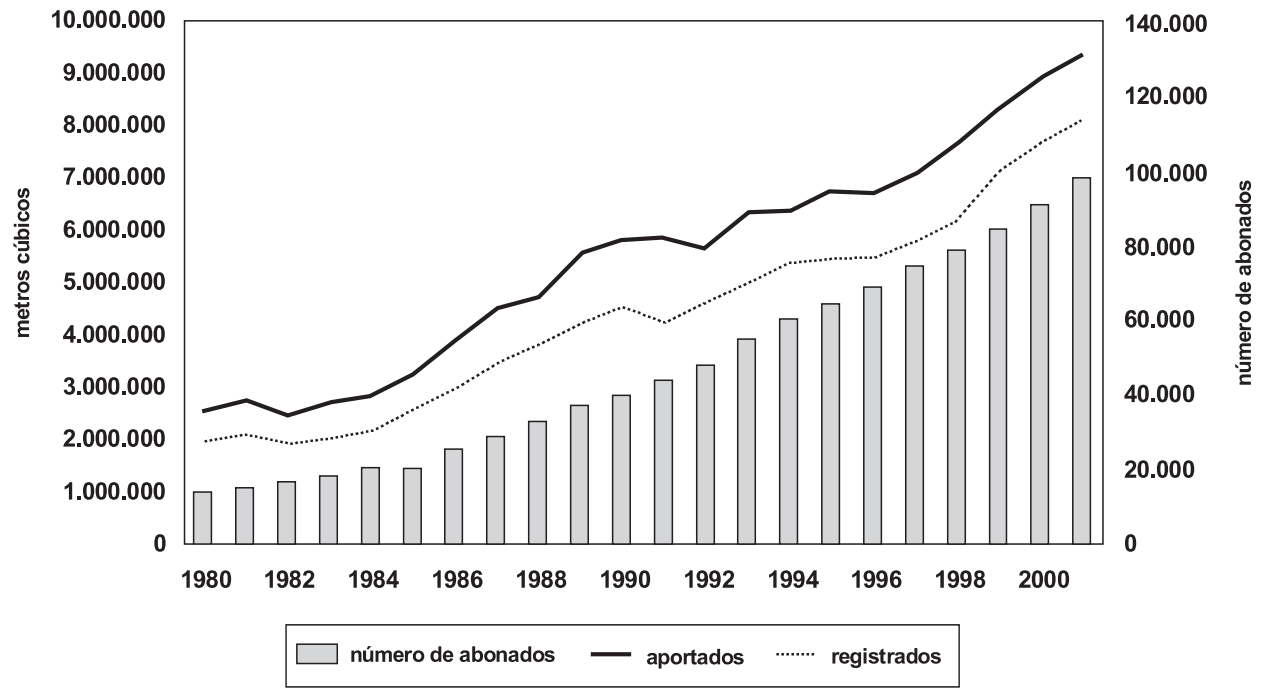

FIGURA 3. Evolución de los caudales aportados y registrados y número de abonados en Torrevieja. Fuente: AGAMED. Elaboración propia

Este factor de eficiencia se traduce en un ahorro en la compra de agua y menor presión sobre el medio cómo un mayor beneficio económico derivado de la reducción de gastos para nuevas captaciones del recurso. Por esto, cabe destacar que las acciones desarrolladas 
por las empresas distribuidoras en baja (Aquagest, Agamed, etc.) y los propios ayuntamientos son eficaces y rentables en sus aspectos económico como social y medioambiental (depuran). Su acción posibilita que se reduzcan costos al encargar el reparto interior domiciliario a la misma empresa gestora que realiza el saneamiento.

La consideración del agua como bien público justificado por la importancia en la actividad humana tiene en su precio una respuesta de considerable valor económico y ambiental. El precio del agua de abastecimiento urbano, procedente del trasvase TajoSegura, aplicado en alta por la MCT a los municipios de la comarca era de $42,10 \mathrm{pts} / \mathrm{m}^{3}$ en el año 2001. Su precio está completamente regulado por el sector público en función de las tarifas del trasvase preferentemente. Sin embargo, el precio aplicado por cada municipio según el nivel de consumo varía significativamente (cuadro no ${ }^{4}$ ).

La característica general es de una respuesta al alza del precio cuyo objetivo es el ahorro y el control del consumo. Un plan de medidas destinadas a crear un modelo de cultura del agua en el que no falte la instalación de nuevas tecnologías en las viviendas a construir en el territorio, cuya implantación es barata y están insuficientemente promovidas e impulsadas en la protección de la naturaleza.

La depuración de las aguas residuales urbanas e industriales en un ecosistema tan frágil como el espacio turístico residencial de la comarca y posterior aprovechamiento, cubre tres objetivos: mantener y mejorar las condiciones medioambientales, incrementar la disponibilidad hídrica en esta zona de escasez de recursos y obtener un beneficio económico y social materializado en el aumento de la riqueza y generación de empleo ${ }^{7}$.

El aprovechamiento como indicador de sostenibilidad fundamenta su criterio en que el agua como factor económico es más importante que el suelo en las tierras de clima árido de la comarca. Por ello, al evaluar la calidad de las aguas depuradas para riego se deben aplicar los mismos criterios que para los recursos superficiales o subterráneos, es decir, la concentración de sales y la presencia de elementos específicos (cloro, sodio, boro, etc.) o peligro de alcalinización. El aprovechamiento es predominantemente para uso agrario con derivaciones hacia parques y jardines y mantenimiento de campos de golf.

Esta nueva forma de utilización de los recursos recuperados sirve de mantenimiento a un nuevo producto turístico (campos de golf) con sentido de estrategia que aumenta la riqueza, genera empleo y no afecta al medio ambiente. Lo mismo que en su aplicación a la expansión o mantenimiento del espacio regado con repercusión socioeconómica de naturaleza semejante. Modelo de seguimiento que tiene en el municipio de Torrevieja el ejemplo más paradigmático del balance entre el volumen de agua consumido $8,8 \mathrm{hm}^{3}$ en el año 2000 y los 5,4 $\mathrm{hm}^{3}$ de agua depurada y de nuevo aprovechamiento. Balance neto de 3,4 $\mathrm{hm}^{3}$, que se traduce, en abonados equivalente, en un consumo de 109 litros por habitante y día. Una manera más práctica y conveniente de analizar el proceso del desarrollo turístico y sus implicaciones económicas, sociales y medio ambientales del territorio turísticoresidencial de los municipios de la comarca de la Vega Baja.

\section{Bibliografía}

BAÑOS, C. (1999): «Modelos turísticos locales. Análisis comparado de dos destinos de la Costa Blanca». Investigaciones Geográficas, no 21 pp. 35-57.

JUÁREZ, C. y VERA, J.F. (1984): «Repercusión económica por cambio de uso y propiedad del agua en el litoral oriolano». Investigaciones Geográficas, no 2 , pp. 139-147.

7 JUÁREZ SÁNCHEZ-RUBIO, C. (1993). La depuración del agua en la mancomunidad de L'Alacantí. Mejora del medio ambiental, repercusión económica y ordenación territorial. Estudios Geográficos no 213, pp. 633-653. 
JUÁREZ, C. (2000): «El papel de la oferta de agua en el desarrollo productivo de la Vega Baja del Segura». Alquibla, no 6, pp. 99-118.

JUÁREZ, C. (2001): «Indicadores hídricos de sostenibilidad y desarrollo turístico en la comarca del Bajo Segura». En Actas del XVII Congreso de Geógrafos Españoles. Oviedo, pp. 354-358.

TORRES, F.J. (1995): Aplicación de un Sistema de Información geográfica al estudio de un modelo de desarrollo local. Crecimiento urbano turístico de Torrevieja. Univeridad de Alicante. 103 p. VERA, J.F. (1987): Turismo y urbanización en el litoral alicantino. Ed. Instituto de Estudios «Juan Gil-Albert». Diputación Provincial de Alicante, 441 p. 